\title{
Features of clinical, radiological and morphological response to preoperative chemotherapy according to biological subtype of breast cancer
}

\begin{abstract}
The aim: to study the correlation of complete clinical and radiological response to preoperative chemotherapy with complete morphological regression in different biological subtype in Russian women with breast cancer (BC) stage T1-3N01-M0.

Materials and methods: Our retrospective study included 213 women with invasive breast cancer stage T1-3N0-1M0 treated in N.N. Blokhin Russian National Cancer Center since 2004 to 2017 treated by preoperative systemic therapy and radical surgery \pm adjuvant therapy. Before chemotherapy all women received clinical examination, mammography, ultrasound, core-biopsy of primary tumor $( \pm 1 \mathrm{ymph}$ node metastases); primary tumor in the breast was clipped with mammography and ultrasound control. At the end of preoperative chemotherapy before radical surgery the clinical examination, mammography and ultrasound was carried out. We compared the clinical and radiological response to preoperative chemotherapy with morphological response according to biological subtype, statistic analyze was made by SPSS 20.0.
\end{abstract}

Results: The response to preoperative chemotherapy significantly depend from the biological subtype and method of evaluation (clinical examination, mammography, ultrasound and pathological assessment $)(\mathrm{p}<0.05)$. The rate of complete pathological response (pCR) in luminal A, luminal B HER2-negative BC, luminal HER2- positive, non-luminal HER2- positive and triple negative BC was $0 \%, 10 \%, 40 \%, 42,3 \%$ and $43,6 \%$, respectively. But the rate of clinical and radiological response, and the proportion of patients with no apparent residual tumor mass in examination of removed breast tissue, including clipped zone, was different, $p<0,05$. So, in patients with luminal HER2- negative BC the results of clinical and radiological assessment of complete response after preoperative systemic therapy are more optimistic than true pCR rate. In luminal A subtype the rate of complete response in clinical examination, mammography, ultrasound was $0-17 \%$, in removing breast tissue in $20 \%$ cases was absent the residual tumor mass, but, in pathological analysis of clipped zone in all women was found residual tumor cells (pCR - 0\%). In luminal B HER2-negative $\mathrm{BC}$ the complete clinical and radiological response was found in $10-15 \%$ women, in removing breast tissue in $28,8 \%$ cases was not found the residual mass, but in microscopic analysis the rate of pCR was $10 \%$ only. In contrast to it, patients with aggressive BC (triple negative and HER2- positive) had underestimation the tumor response: the rate of complete clinical and radiological response in women with triple negative $\mathrm{BC}$ was $31-42 \%$, and in $28,2 \%$ cases only in removing breast tissue was not found the residual tumor, but the rate of true $\mathrm{pCR}$ was significantly higher $(43,6 \%)$. In women with non- luminal HER2- positive BC the rate of clinical and radiological response was $32-42 \%$, in removing breast tissue in $26,9 \%$ was not found the residual mass, but in microscopic analysis the rate of $\mathrm{pCR}$ in clipped zone was significantly higher $(42,3 \%)$.

Conclusion: in patients with $\mathrm{BC}$ stage $\mathrm{T} 1-3 \mathrm{~N} 0-1 \mathrm{M} 0$ received preoperative systemic therapy the rate of $\mathrm{pCR}$ significantly depend from biological subtype and methods of tumor response assessment.

Keywords: breast cancer, tumor, preoperative systemic therapy, clinical, mammography, ultrasound, MRI
Volume 5 Issue 3 - 2018

\author{
Kolyadina Irina Vladimirovna,' Pavlikova Olga \\ Arkadievna,' Bokhian Vagan Yurikovich,' \\ Danzanova Tatiana Yurievna, ${ }^{3}$ Karpova Marina \\ Sergeevna, ${ }^{4}$ Kozlov Nikolai Alexandrovich, ${ }^{5}$ \\ Poddubnaya Irina Vladimirovna ${ }^{6}$ \\ 'Department of Russian Medial Academy of Continuing \\ Professional Education on the base of N.N. Blokhin National \\ Cancer Research Center, Russia \\ ${ }^{2}$ Head of Surgical Department of Tumor Diagnostic, N.N. \\ Blokhin National Cancer Research Center, Russia \\ ${ }^{3}$ Leading Researcher of the Department of Radiation \\ Diagnostics, N.N. Blokhin National Cancer Research Center, \\ Russia \\ ${ }^{4}$ Department of Radiation Diagnostics, N.N. Blokhin National \\ Cancer Research Center, Russia \\ ${ }^{5} \mathrm{Head}$ of the Pathology Department, N.N. Blokhin National \\ Cancer Research Center, Russia \\ ${ }^{6} \mathrm{Head}$ of Oncology Department of Russian Medial Academy of \\ Continuing Professional Education, on the base of N.N. Blokhin \\ National Cancer Research Center, Russia
}

Correspondence: Kolyadina Irina Vladimirovna, Department of Russian Medial Academy of Continuing Professional Education on the base of N.N. Blokhin National Cancer Research Center, Moscow, Russia, Email irinakolyadina@yandex.ru

Received: April 29, 2016 | Published: June 14, 2018

\section{Introduction}

Breast cancer (BC)-the most common cancer pathology in the world. In Russia, in 2017, more than 68 thousand new cases were found, in $67 \%$ Russian women the diagnosis was established in stage II-III, in which it is advisable to carry out preoperative systemic therapy. ${ }^{1,2}$ The most important goal of preoperative systemic therapy is a complete pathological response (pCR), since this factor is associated with high rate of survival, so, morphological evaluation of pathological response is given great importance all over the world. ${ }^{3-6}$ However, quality assessment of pathological response is possible only targeted interventional marking of primary tumor and regional lymph nodes used before chemotherapy start. The clips are the only reference point of the tumor bed at the stage of surgery and morphological evaluation of the therapy result. ${ }^{7-9}$ 
In clinical practice the effectiveness of preoperative systemic therapy is studied by different methods (clinical, mammography, ultrasound, MRI), but the most precise accurate and important method is the pathological response evaluation of clipped zone of primary tumor bed and lymph node metastases. ${ }^{10-13}$ However, there is no reliable data, do the clinical and radiological data after preoperative chemotherapy correspond to complete morphological regression in different biological subtype.

\section{Propose}

To study the correlation of complete clinical and radiological response to preoperative chemotherapy with complete morphological regression in different biological subtype in Russian women with breast cancer stage T1-3N01-M0.

\section{Material and methods}

The study included 213 women with invasive breast cancer stage T1-3N0-1M0 treated in N.N. Blokhin Russian National Cancer Center since 2004 to 2017. Standard plan of examination in all women before begining of preoperative systemic therapy included clinical examination, mammography, ultrasound, core-biopsy of primary tumor ( \pm lymph node metastases) with morphological assessment histological type, grade of tumor and receptor status (expression of ER, PR, HER2 and Ki67). At the end of preoperative chemotherapy before radical surgery the clinical examination, mammography and ultrasound was carried out. All patients had the radical surgery (breast-conserving, $33,3 \%$ or mastectomy, $66,7 \%$ with standard axillary lymph node dissection) with morphological evaluation of tumor response. For clinical and radiological assessment of tumor response after preoperative chemotherapy standard WHO criteria were used (complete response, partial response and stabilization). Morphological assessment considered the complete response (pCR) or the presence of residual tumor by microscopic data. We compared clinical and radiological response to preoperative chemotherapy with morphological response according to biological subtype, statistic analyze was made by SPSS 20.0 .

\section{Results}

\section{Patient and tumor characteristics}

Median age of Russian women $(\mathrm{n}=213)$ included in our study was 47 years (range 24-76). Breast cancer stage distribution was: stage T1N1M0 -3,3\%, T2N0M0-18,8\%, T2N1M0- 44,6\%, T3N0M0-7,0\%, T3N1M0-26,3\%. All patients had an invasive breast cancer; ductal cancer was dominated $(92,6 \%$ cases $)$, lobular cancer was seen in $4,5 \%$ and other histological type had $3 \%$ women only. Tumor grade distribution was: G1-7,8\%, G2-67,7\%, G3- 24,6\%.

Biological subtype of primary tumor was known in 192 patients; luminal A-in 7,8\% cases, luminal B HER2-negative-in 42,2\%, luminal HER2-positive-in $16,1 \%$, non-luminal HER2+positive-in $13,5 \%$ and triple negative breast cancer was found in $20,3 \%$ patients.

\section{Preoperative systemic therapy}

Preoperative systemic therapy received all women; chemotherapy-204 women, endocrinotherapy- 9 patients. Preoperative chemotherapy included treatment regimens with anthracyclines (AC, FAC, CAF)-in $49,5 \%$ patients, taxanes in monotherapy-in $2,5 \%$, combination of anthracyclines and taxanes $-30,4 \%$, combination of anthracyclines, taxanes and platinum- $4,9 \%$, combination taxanes and platinum-11,8\%, and in $1 \%$ cases used combination anthracyclines and platinum. In neoadjuvant chemotherapy of HER2+ breast cancer trastuzumab was used $(78,9 \%)$ or dual anti-HER2 blockade (trastuzamab and pertuzumab)-in 12,3\% cases. Median number of cycles of neoadjuvant chemotherapy was 6 (range 1-8). Neoadjuvant endocrinotherapy received 9 women with luminal HER2- negative BC; 2 women - tamoxifen, and 7 women - aromatase inhibitors.

\section{Clinical, mammography and ultrasound response evaluation according to biological subtype}

Size of primary tumor was measured before and after preoperative systemic therapy with clinical examination, mammography and ultrasound in all women. Before systemic therapy according to clinical data the size of the primary tumor was $2-10 \mathrm{sm}$ (mean- $4,3 \mathrm{sm}$, median- $4 \mathrm{sm}$ ), the tumor volume was $4,5-720 \mathrm{sm}^{3}$ (mean- $68,1 \mathrm{sm}^{3}$, median- $\left.32 \mathrm{sm}^{3}\right)$. According to mammography the tumor size was $2-11 \mathrm{sm}$ (mean 3,6sm, median-3sm), the tumor volume was $3-945 \mathrm{sm}^{3}$

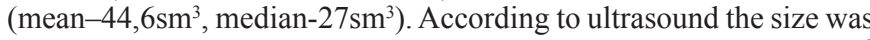
$2-7 \mathrm{sm}$ (mean-3,2sm, median-3sm), the tumor volume was $1-119 \mathrm{sm}^{3}$ (mean-23,1 sm3, median- $15,5 \mathrm{sm}^{3}$ ).

After preoperative chemotherapy complete clinical response was seen in $27,8 \%$ women, complete mammography response-in $21,5 \%$, complete ultrasound response-in $29,7 \%$ patients; so, residual tumor in the breast was found in clinical examination, mammography and ultrasound in $72,2 \%, 70,3 \%$ and $78,5 \%$ cases. After preoperative systemic therapy according to clinical data the size of the residual tumor was $0-6 \mathrm{sm}$ (mean- $1,9 \mathrm{sm}$, median-2sm), the tumor volume was $0-151 \mathrm{sm}^{3}$ (mean-13,3 $\mathrm{sm}^{3}$, median-6 $\left.\mathrm{sm}^{3}\right)$. According to mammography the tumor size was $0-8 \mathrm{sm}$ (mean $2,1 \mathrm{sm}$, median-2sm), the tumor volume was $0-263 \mathrm{sm}^{3}$ (mean-15,3 $\mathrm{sm}^{3}$, median- $4 \mathrm{sm}^{3}$ ). According to ultrasound the size was $0-5 \mathrm{sm}$ (mean-1,5sm, median-1 sm), the tumor volume was $0-64 \mathrm{sm} 3$ (mean-4,5 $\mathrm{sm}^{3}$, median- $1 \mathrm{sm}^{3}$ ).

According to clinical examination, the clinical complete response was seen in $29,1 \%$ patients; in liminal A subtype-in $8,3 \%$ only, in luminal B HER2-negative-in 15,4\%, in luminal HER2- positive-in $48,1 \%$, in non-luminal HER2- positive-in $32 \%$ and in triple-negative BC - in $30,6 \%$ cases, $p=0,001$ (Table 1 ).

According to mammography, the radiological complete response was absent in women with luminal A subtype $(0 \%)$ but was stated in 9,5\% cases in luminal B HER2-negative BC, in luminal HER2positive-in $44,4 \%$, in non-luminal HER2- positive-in $20 \%$ and in triple-negative $\mathrm{BC}-$ in $41,7 \%$ cases, $\mathrm{p}=0,003$ (Table 1 ).

According to ultrasound, the radiological complete response was seen in $31,6 \%$ cases; in liminal A subtype-in $16,7 \%$, in luminal B HER2-negative-in $9,7 \%$, in luminal HER2-positive-in $56,2 \%$, in non-luminal HER2- positive-in $42,1 \%$ and in triple-negative BC-in $39,1 \%$ cases, $p=0,009$.

\section{Pathological response evaluation}

The pathology assessment of tumor response included 2 steps: examination of breast tissue for residual tumor and microscopic analyze of this zone to determinate the degree of morphological response. In examination of breast tissue, the residual tumor was found in $71,6 \%$ women, medial tumor volume of the residual tumor mass was $3 \mathrm{sm}^{3}\left(0-309 \mathrm{sm}^{3}\right)$. In $28,4 \%$ patients in examination of breast tissue the pathologist did not find the residual tumor mass, including clipping zone; in $20 \%$ cases-in liminal A subtype, in $28,8 \%$-in luminal B HER2-negative BC, in 33,3\% - in luminal HER2-positive, in 26,9\%- non- luminal HER2-positive and in $28,2 \%$ women with triple negative breast cancer, $\mathrm{p}=0,923$.

Second step of morphological assessment was the microscopical analyze of pathological response; $\mathrm{pCR}$ was seen in $22,7 \%$ cases. The most interesting, that was the discordance between absent of residual tumor mass in the breast and true pCR rate in microscopic analyze. So, in patients with luminal A subtype in $20 \%$ cases there was no the residual tumor zone in the breast in clipped zone, but in microscopic 
analyze was found residual breast cancer cell in all cases. In women with luminal B HER2-negative BC in $28,8 \%$ cases the residual tumor was absent, but in microscopic analyses true pCR had $10 \%$ women only. In patients with HER2+BC the frequency of absent of residual tumor in macro- and microscopic analyze was a very similar $(33,3$ and $40 \%)$. But in women with non-luminal HER2-positive and triple negative $\mathrm{BC}$ were seen a very interesting phenomenon: the absence of a residual tumor zone in breast examination by pathologist was noted significantly less often than detection of true rate of $\mathrm{pCR}$ in microscopic analyze (in non- luminal HER2- positive BC-26,9\% vs $41,3 \%$, respectively; in triple negative $\mathrm{BC}-28,2 \%$ vs $43,6 \%$, respectively), $p=0,001$, Table 1 .

Table I Clinical, radiological and morphological evaluation of tumor response after preoperative systemic therapy according to biological subtype

\begin{tabular}{|c|c|c|c|c|c|c|}
\hline & \multicolumn{5}{|c|}{ Biological subtype of BC/number patients (\%) } & Patients \\
\hline & \multicolumn{5}{|c|}{ I-luminal A } & $\mathbf{N}(\%)$ \\
\hline & \multicolumn{6}{|c|}{ 2- luminal B HER2- negative } \\
\hline \multirow[t]{4}{*}{ Method of response evaluation } & \multicolumn{6}{|c|}{ 3- luminal HER2- positive } \\
\hline & \multicolumn{6}{|c|}{ 4- non-luminal HER2- positive } \\
\hline & \multicolumn{6}{|c|}{ 5- triple negative } \\
\hline & $\mathbf{I}$ & 2 & 3 & 4 & 5 & \\
\hline \multirow{2}{*}{$\begin{array}{l}\text { Clinical examination, complete } \\
\text { response } p \text {, value }\end{array}$} & $\mathrm{I}(8,3 \%)$ & $10(15,4 \%)$ & |3(48, | \%) & 13(32\%) & $I(30,6 \%)$ & $48(29,1 \%)$ \\
\hline & 0,001 & & & & & \\
\hline \multirow{2}{*}{$\begin{array}{l}\text { Mammography, complete } \\
\text { response } \mathrm{p} \text {, value }\end{array}$} & $0(0)$ & $4(9,5 \%)$ & $8(44,4 \%)$ & $4(20 \%)$ & $10(41,7 \%)$ & $26(23,2 \%)$ \\
\hline & 0,003 & & & & & \\
\hline \multirow{2}{*}{$\begin{array}{l}\text { Ultrasound, complete response } \\
\text { p, value }\end{array}$} & $\mathrm{I}(16,7 \%)$ & $3(9,7 \%)$ & $9(56,2 \%)$ & $8(42,1 \%)$ & $9(39,1 \%)$ & $30(31,6 \%)$ \\
\hline & 0,009 & & & & & \\
\hline \multirow{2}{*}{$\begin{array}{l}\text { No residual mass in removing } \\
\text { breast tissue } p \text {, value }\end{array}$} & $3(20 \%)$ & $23(28,8 \%)$ & $10(33,3 \%)$ & $7(26,9 \%)$ & II $(28,2 \%)$ & $54(28,4 \%)$ \\
\hline & 0,923 & & & & & \\
\hline \multirow{2}{*}{$\begin{array}{l}\text { Pathological complete response, } \\
\text { pCR p, value }\end{array}$} & $0(0)$ & $8(10 \%)$ & $12(40 \%)$ & II (42,3\%) & $17(43,6 \%)$ & $48(25,3 \%)$ \\
\hline & 0,001 & & & & & \\
\hline
\end{tabular}

\section{Discussion}

Complete pathological response (pCR) after preoperative systemic therapy is the subject to favorable prognosis in aggressive biological subtype (triple negative and HER2-positive) and associated with high rate of long-term survival, so preoperative systemic therapy has become a priority initially step of treatment not only locally advanced breast cancer, but also early stage. ${ }^{2-6}$ The evaluation of the tumor response to preoperative systemic therapy includes the clinical examination, mammography, ultrasound and pathological assessment of the removed breast tissue. It is a very important to clip the tumor before starting systemic therapy because it allows to find the tumor bed after preoperative systemic therapy, at the stage of surgery and morphological study. ${ }^{11,12}$ The volume of radical surgery (mastectomy or breast- conserving surgery) is planned into account the tumor response which is estimated by clinical examination, mammography and ultrasound. ${ }^{3,8,9}$ In step of morphological analysis of tumor response considers not only the presence of the residual tumor tissue, but also it characteristics and degree of damage of tumor cells. But the accordance between total clinical and radiological response and established fact of pCR in the microscopic examination of removal breast tissue according to biological subtype are not enough studied. ${ }^{14-18}$ In our retrospective analysis we found that the rate of tumor response after preoperative chemotherapy in Russian depended not only on the biological subtype, but also on the method of assessing the response (clinical examination, mammography, ultrasound or pathological evaluation).

Only in women with luminal HER2- positive BC the result of the clinical and radiological evaluation coincides with the data of microscopic analysis; but in patients with luminal HER2-negative $\mathrm{BC}$ the results of clinical and radiological assessment of complete response after preoperative systemic therapy are more optimistic than true pathological complete response in microscopic analysis. In contrast to it, patients with aggressive biological subtype of BC (triple negative and non-luminal HER2-positive) had underestimation the tumor response at the clinical and radiological examination. The rate of complete clinical and radiological response in aggressive $\mathrm{BC}$ was significantly lower than the rate of true pCR in microscopic analysis. Apparently, in aggressive non-luminal cancer (HER2+ or triple negative) the tumor regression during chemotherapy occurs with a pronounced microenvironment reaction and the formation of the coarse fibrosis, which is perceived as a residual tumor in the clinical and radiological evaluation but in microscopic analysis reveals the tumor cells. This phenomenon of the tumor regression in different biological subtype $\mathrm{BC}$ after preoperative systemic therapy is unique and requires further study. 


\section{Clinical case}

Patient 62 years old. Breast cancer right cT2N0M0 (invasive ductal cancer, G3, ER-0 PR-0 HER2-0 Ki67- 75\%). Preoperative chemotherapy (docetaxel with carboplatin, 6 cycles), before chemotherapy primary tumor in the breast was clipped. Clinical and radiological assessment of tumor response: small residual tumor in the breast in the clipped zone (Figure 1). Before radical breastconserving surgery the residual tumor was marked by needle-guide, mammography control of removal breast tissue was made (Figure 2). In microscopic analysis of clipped tumor bed-complete pathological response, pCR (Figure 3).

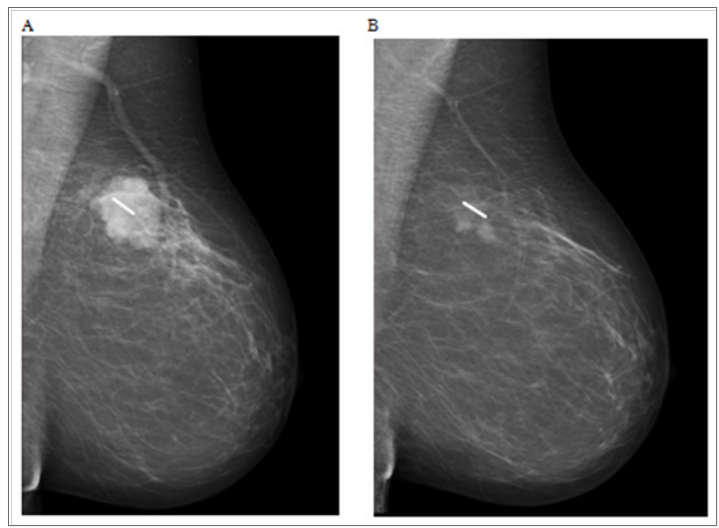

Figure I Mammography control after marking the tumor before chemotherapy (A) and after chemotherapy (B).

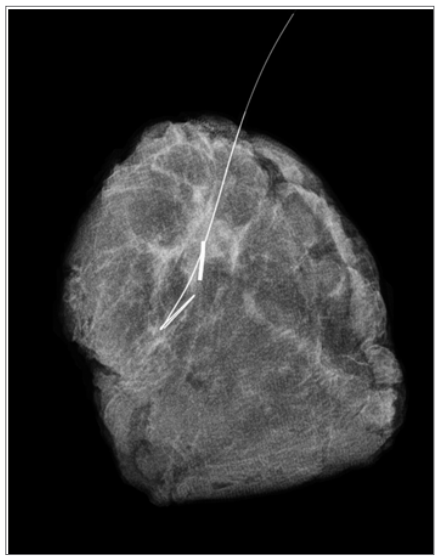

Figure 2 Mammography control of clipped tumor zone in the removing breast tissue.

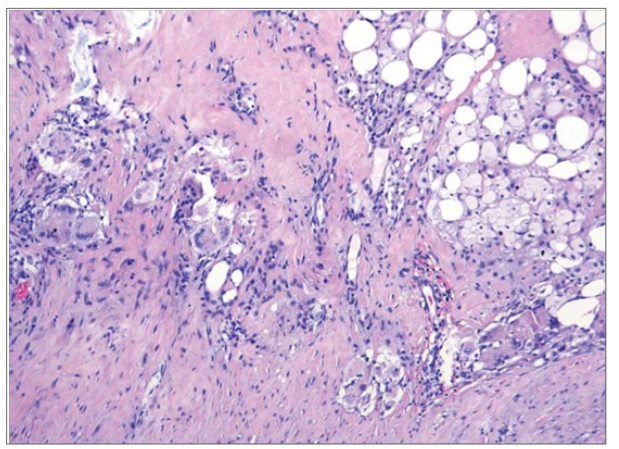

Figure 3 Complete pathological response, pCR in the clipped tumor bed.

\section{Acknowledgements}

None.

\section{Conflict of interest}

The author declares that there is no conflict of interest.

\section{References}

1. http://www.oncology.ru/service/statistics/malignant_tumors/2016.pdf

2. https://rosoncoweb.ru/standarts/RUSSCO/2017/recoms2017_08.pdf

3. Wolmark N, Wang J, Mamounas E. Preoperative chemotherapy in patients with operable breast cancer: nine-year results from National Surgical Adjuvant Breast and Bowel Project B-18. J Natl Cancer Inst Monogr. 2001;(30):96-102.

4. Cortazar P, Zhang L, Untch M, et al. Pathological complete response and long-term clinical benefit in breast cancer: The CTNeoBC pooled analysis. Lancet. 2014;384(9938):164-172.

5. Coates AS, Winer EP, Goldhirsch A, et al. Tailoring therapies-improving the management of early breast cancer: St Gallen International Expert Consensus on the Primary Therapy of Early Breast Cancer 2015. Ann Oncol. 2015;26(8):1533-1546.

6. Corben AD, Abi-Raad R, Popa I. et al. Pathologic response and longterm follow-up in breast cancer patients treated with neoadjuvant chemotherapy: a comparison between classifications and their practical application. Arch Pathol Lab Med. 2013;137(8):1074-1082.

7. Kolyadina IV, Poddubnaya IV, Pavlikova OA, et al. The evolution of neoadjuvant approach in primary operable breast cancer last decade: modern trend or a real clinical practice? The evolution of neoadjuvant approach in primary operable breast cancer last decade: modern trend or a real clinical practice? Journal of Modern Oncology. 2017;19(1):9-16.

8. Carey LA, Metzger R, Dees EC, et al. American joint committee on cancer tumor-node-metastasis stage after neoadjuvant chemotherapy and breast CAncer outcome. J Natl Cancer Inst. 2005;97(15):1137-1142.

9. Criscitiello C, Azim HA, Agbor-tarh D, et al. Factors associated with surgical management following neoadjuvant therapy in patients with primary HER2- positive breast cancer: results from the NeoALTTO phase III trial. Ann Oncol. 2013;24:1980-1985.

10. Gu YL, Pan SM, Ren J, et al. Role of Magnetic Resonance Imaging in Detection of Pathologic Complete Remission in Breast Cancer Patients Treated With Neoadjuvant Chemotherapy: A Meta-analysis. Clin Breast Cancer. 2017;17(4):245-255.

11. Kolyadina IV, Poddubnaya IV, Pavlikova OA, et al. Features of surgical treatment of patients with breast cancer receiving preoperative systemic therapy. Journal of Modern Oncology. 2016;18(1):50-54.

12. Youn I, Choi SH, Kook SH, et al. Ultrasonography-guided surgical clip placement for tumor localization in patients undergoing neoadjuvant chemotherapy for breast cancer. J Breast Cancer. 2015;18(1):44-49.

13. Anreeva Yu, Moskvina LV, Berezina TA, et al. Procedure for intraoperative material examination in breast cancer after neoadjuvant therapy to estimate residual cancer burden using the RCB system. Archivas of pathology. 2016;78(2):41-46.

14. Pavlikova OA, Kolyadina IV, Komov DV, et al. Predictive factors of achievement pathological complete response at neoadjuvant chemotherapy of primary operable breast cancer. Journal of Modern Oncology. 2017;19(1): 24-29.

15. Lee HJ, Park IA, Song IH, et al. Comparison of Pathologic Response Evaluation Systems after Anthracycline with/without Taxane-Based Neoadjuvant Chemotherapy among Different Subtypes of Breast Cancers. 


\section{PLoS One. 2015;10(9):e0137885}

16. Heil J, Schaefgen B, Sinn P, et all. Can a pathological complete response of breast cancer after neoadjuvant chemotherapy be diagnosed by minimal invasive biopsy? Eur J Cancer. 2016;69:142-150.

17. Menes TS, Golan O, Vainer G, et al. Assessment of Residual Disease With Molecular Breast Imaging in Patients Undergoing Neoadjuvant Therapy: Association With Molecular Subtypes. Clin Breast Cancer. 2016;16(5):389-395.
18. Schaefgen B, Mati M, Sinn HP, et al. Can Routine Imaging After Neoadjuvant Chemotherapy in Breast Cancer Predict Pathologic Complete Response? Ann Surg Oncol. 2016;23(3):789-795. 